\section{LES ÉTATS-UNIS PENDANT LA GUERRE}

Nous extrayons du Bulletin de la Chambre de Ciommerce française de New-York de juillet 1918, les intéressants renseignements et jugements suivants.

On appréciera notamment l'opportunité des mesures a prenare, à la veille de la conclusion des traités de commerce qui vont suivre la signature de la Paix, pour mettre l'Allemagne dans l'ımpossibilité de recommencer ses métaits.

\section{I. - LE MATÉRIEL DE GUERRE IMPORTÉ DES ÉTATS-UNIS}

Le département fédéral du Commerce a publié le rapport annuel consacré aux importations et aux exportations des produits qui servent aux transactions des Etats-Unis.

Les empires du centre, Allemagne et Autriche, ne som plus autorisés à faire du commerce. avec ce pays ; la Bulgarie et la Turquie, auxquelles le Gouvernement n'a pas declaré la guerre, ne peuvent pas faire avec elles grand trafic,

La plupart des alfaires se font avec les Alliés et on éprouve de gros embarras à travailler avec les neutres, trop sujets à caution, et qui fournissent souvent à nos ennemis des produits que l'on ne veut pas leur vendre directement.

L'Angleterre, avant l'entrée en guerre des Etats-Unis, a eu d'immenses difficultés à surmonter pour empècher l'Allemagne de recevoir les marchandises dont elle avait un pressant besoin, comme le cuivre, certains autres métaux et le caoutchouc, les graisses et les huiles de graissage, etc. Une de ces grandes difficultés, même après la décläration de guerre, a été l'appui de tous les moments donné par les Allemands en résidence aux Etats-Unis à leurs compatriotes d'Europe, soit parce qu'ils y sont très nombreux, soit parce qu'ils sont aidés dans leur vilaine besogne par des Américains que l'on n'aurait pas soupçonné d'être leurs partisans. Heureusement les flottes anglaises ont réussi, dan's bien des cas, à retenír les marchandises américaines envoyées en Allemagne par des chemins détournés, tandis que celles achetées ici par les Alliés ont pu être transportées aux ports indiqués par leur Gouvernement.

En 1914 , les cargaisons de munitions de guerre ont atteint la somme de $\$ 17.779 .000$. Elles comprenaient des armes à feu, des poudres et áutres explosifs, des fils de fer et des pièces d'aéroplanes et sous le nom d'aćcessoires - Ies automobiles, motocycles, appareils scientifiques, très demandés. Les Etats-Unis en ont envoyé pour \$36.1 82.000 .

Les équipages et équipements et les produits chimiques, les chevaux et les mules pour la cavalerie et les transports, formant une catégorie à part, les machines outils et les métaux paraissent dans le rapport du Ministère sous leurs diverses rubriques représentant au totàl : \$2 I 6 .I 82.000 .

Les cnvois totalisés des munitions et équipements nécessaires à la conduite des hostilités, ont été pendant la première année fiscale de guerre en rgr4, de $\$ 270$. jo2.00o. Ils furent presque doublés, l'année suivante, avec un total de $\$$ 435.003.00o. Ils comprenaient pour $\$ 66.857 .000$ de munitions de guerre, $\$ 27.033 .000$ d'automobiles, de motocycles et d'instruments scientifiques divers ; les chevaux et mulets, les métaux. les machines outils, les chaussures et les cuirs, équipements, etc., pour $\$ 296 . \times 3.000$.

L'année suivante, $19 \mathrm{r} 6$, les munitions de guerre ont atteint le chiffre considérable de $\$ 532$. r ro.ooo, et celui de la deuxième catégorie, les accessoires, $\$$ I.29.237.000. Pour la troisième classe, la somme dépensée a atteint \$668. i i r.ooo, le tout a fait un total de $\$$ r.329.458.0oo.
En 1917, le total des cargaisons reçues en France, pour la gucrue proprement dite, a été de \$2.127.940.000, composć de $\$ 328.563 .000$ de munilions, de $\$ 100.385 .000$ d'accessoires et d'environ $\$$ r.og8.392.0oo de fournitures diverses, tous articles absolument indispensables à la guerre.

En outre de ces marchandises envoyées en France et en Angleterre, et pointées par le Département du Commerce, le Comité du commerce de guerre fait embarquer pour les troupas des Etats-Unis et pour la marine, des quantités considérables de produits divers, de munitions et d'équipement qui ne sont pas portées, pour le moment, du moins, sur les registres de sortie dudit département. Ces cargaisons sont incrites dans un service spécial qui fait partic de l'intendance et qui n'a trait qu'à la guerre et à l'approvisionnement des soldats américains. Il est naturel que les chiffres de ces exportations ne soient point publiés maintenant, afin de ne pas servir de bases pour les calculs des états-majors allemands, indication qui leur serait prérieuse et qu'ils connấtraient de suite par leurs espions: - Les renseignements qu'ils obtiennent sont déjà trop importants !...

La police américaine trouve de temps en temps des appareils de télégraphie sans fil installés près des grèves ou dans les grands bois de l'intérieur, mais les juges ne sont pas assez sévères ; on se contente généralement de mettre les coupablès de ces trahisons dans l'impossibilité de nuire en les envoyant dans les camps de concentration.

\section{II. - LE COMMERCE DE L'ALLEMAGNe D'AVANT-GUERRE}

Les nations, obligées de trouver les ressources nécessaires au bon fonctionnement de leur Gouvernement, ont dû depuis bien longtemps organiser les douanes. Suivant le caractère des habitants, cette institution est plus ou moins chicanière, processive, et soupçonneuse. En outre, dans certaines localités, les employés de la douane dolvent accepter les suggestions des concurrents dans la conduite de leur service et telle marchandise qui entrerait sans difficulté en payant les droits d'un tarif connu, est retenue et lon ergote pendant des semaines avant de la laisser passer sur les marchés qui attendent.

De plus, on a fait de la douane une arme dangereuse pour les compétiteurs. L'Allemagne notamment s'efforçait de son mieux à arrêter aux frontières les marchandises, et surtout les vins français. Elle avait imaginé des analyses à faire dans ses laboratoires et l'on sait que l'honnêteté de ses fonctionnaires est loin d'être reconnue sans tache. On peut penser quelles difficultés les chimistes teutons élevaient contre ces vins français dont leur pays est si jaloux 1... Le public des, Etats-Unis a maintenant devant les yeux un résumé des actes hónteux commis par l'Ambassadeur de cette nation de barbares, et le proverbe français est toujours vrai qui dit : "Tel maître, tel valet ".

La guerre qui a été déclarée par l'Allemagne, est avant tout une Guerre de Commerce. Ces gens, fort orgueilleux depuis leurs victoires contre l'Autriche, leurs divers agrandissements de territoures volés à leurs voisins et finalement leur guerre de 1870 , qui a été préparée par Bismarck, au moyen d'un faux, comme celle de rgr4, ont pensé qu'ils allaient devenir les maîtres du monde. Après avoir, pendant quarante ans, façonné leurs sujels àr leur image, les Hohenzollern et leurs partisans ont lancé leurs soldat à la conquête du Monde. Ils avaienl d'abord cherché à s'emparer pacifiquement du commerce européen et américain ;! ils avaien! poussé leurs tentacules de pieuvre jusqu'en Australie, où ils avaient presque réussi à contrôler les métaux, comme aussi aux Ftats-Unis et un peu, partout 'Ils vendaient à leurs peuples les marchandises de leur fabrication, plus cher qu'aux étrangers. pour ruiner les usines et les fabriques de ceux qui, bonnement. les recevaient chez eux. Ils avaient si bien manouvré que, même en France, où l'on aurait cependant dû les connaître, on préférait donner du travail à un Allemand, pour laisser les enfants du pays s'expatrier afin de gagner la vie de leur famille en exil. Partout 
en France et dans les colonies, l'on trouvait d'énormes quantités de marchandises allemandes, faisant une concurrence désastreuse aux produits français.

Cétte mème émigration se répandart aussi en Italıe, en Angleterre, en Belgıque et presque dans toute l'Europe. En Itahe, on ne voyait que des maisons allemandes et on entendat parler allemand dans tout le pays, en Angleterre, c'était un paradis pour tous ces jeunes.gens que la douce Allemagne envoyait dans les villes commerçantes de lîle. Elle ne se contentait pas de pousser ses fils à l'étranger, elle y faisait pénétrer ses filles qu y devenalent institutrices, femmes de chambre ou domestiques, toujours prêtes à épier et à sausir un secrel. Ces demoisellés travaillaient de préférence chez les officiers des armées du pays qui avail eu le plaisir de les recevoir.

Cela a duré longtemps ; l'Allemand est patient. Mais quelques uns des chefs du pays ne l'étaient pas ct ils ont voulu aider la pénétralion pacifique en déchaînant la guerre qui, à leur dire, ne devait pas durer plus de six mois. Ils voulaient absolument dominer le Monde et imposer leur joug pesant sur l'Europe et sur l'Amérique, puis enchaîner l'Asie el rassasier avec les dépoulles leur appétit vorace en prenant les Indes.

Le principal objet de l'Allemagne était et est encore de détruire le commerce. En effet, ses armées ont pillé les usines et tous les moyens de production en Belgique, dans le nord de la France, en Serbie et en Roumanie, partout enfin, où les barbares ont réussi à pénétrer. Sa marine bloquée par l'Angleterre n'a jamais pu sortır sans être battue piteusement, mais elle a envoyé ses sousmarins, criminels, qui ne peuvent combattre à ciel ouvert, pour torpiller non seulement les navires de ses ennemis avérés, maıs ceux des pays neutres dont les gouvernements accèptent sans mot dire ces étonnantes 'opérations maritimes.

L'Allemagne, après de nombreuses années de preparation, détruit les vaisseaux neutres qui ne sont pas engagés dans le commerce avec les belligérants, afin de réduire le tonnage des llottes du Monde, et d’accroître par les pertes infligées aux autres nations, ses propres flottes qu'elle conserve pour l'après-guerre. Par ce moyen, elle pourra augmenter son commerce avec les neutrés et en même temps, causer beaucoup de tort aux nations qui sont ses ennemies. Ainsi, elle croit 'qu'elle pourra fortifier sa puissance industrielle et commerciale. En dépouillant les pays limitrophes de leurs richesses et de leur pouvoir, elle étendra son emprise sur eux, les emploiera à s'enrichir et arrivera à ses fins. Cetle sorte de calcul de la part de l'Allemagne va se continuan: et sadresse non seulement aux pays affaiblis qui l'entourent et aux régions de l'Est, mais encore à la Grande Bretagne et au continent de l'ouest dont elle voudrait circonscrire les relations industrielles et commerciales.

G'est peut-être le désir des Empires du Centre de chercher à reprendre la tête du commerce du Monde, mais les Allemands auront fort à faire pour reconstituer leur puissance. Ils ont fait trop de mal inutile ; leur conduite de la guerre a été si inhumaine, si sauvage qu'ils'se sont attiré la haine de tous les peuples civilisés. Les Alliés détiennent les matières premières qui sont nécessaires aux fabriques teutonnes et ils ne les laisseront pas ressaisir par leurs ennemis présents. Le premier minitre d'Angleterre a déclaré qu'un accord éntre les Alliés " signifíe que le sort économique du monde sera entre les mains des. grandes puissances alliées ensemble dans la guerre actuelle ", et il espère que l'Angleterre et les Etats-Unis seront absolument d'accord sur le problème économique comme sur les autres.

PROCESS VERBAUX DE LA COMMISSION DES FORCES HYDRAULIQUES

L'abondance des matières nous oblige à reporter aux numéros suivants la continuation de ces Procès-Verbaux dont la publication approche de sa fin. La discussion du Projet de loi dont elles s'occupent n'étant pas encore ouverte, leur place sera momentanément cédée àdes communications de plus d'actualité. Noslecteurs, pensons-nous, admettront aisément cette disposition.
REVUE DES SOCIÉTÉS SAVANTES ET DES PUBLICATIONS SCIENTIFIQUES

\section{ACADÉMIE D'AGRICULTURE Séance du 26 Décembre 191\%}

\section{APPLICATION DE L'ÉLECTRICITÉ A LAGRICULTURE}

M. DaBat, chargé par la Section de Génie rural de licadémie, d'examiner une étude de M. Lours Martin sur les moyens d applquer lélectricité à l'agriculture, a complété les observations technıques présentées dans son rapport en donnant des renseignements sur limportance actuelle des emplois de l'électricilé en agriculture et en indiquant les mesures qui paraissent de nature à contribuer au développement des utilısations agricoles de l'énergie. Après un bref résumé du développement des utilisations de nos forces hydrauliques, le rapport indique les diverses mesures d'ordre technuque, administratif et législatif prises par le Ministre de l'Agriculture pour favoriser les emplors agricoles de toute nature.

Dans le domaine adminıstraulf el législatif, le Ministère de l'Agriculture a tracé les règles à observer par les Communes dans la cession de leurs droits de riveraincté, el à côté des mesures qui tendent à diminuer le prix de la force par l'abaissement des prix de revient, il s'est occupé de faire rédure directement les tarifs applicables aux opérations agricoles.

Dans cet ordre d'idées, l'Administration malgré des résistances difficiles à vaincre a pu introduire dans les cahiers des charges de concession de distribution d'énergie, le principe de la réduction de tarifs en faveur des entreprises réalisées par des associations syndicales. Elle a également obtenu que l'obligation de fournitures à bas prix soit prévue dàns diverses concessions d'usines sur les rivières du domaine public, accordées par le Ministre des Travaux publics.

Flargissant la question et considérant que les avantages prévus en faveur, de l'industrie pour l'établissement des usines doivent, dans l'intérêt général, avoir pour contre-partie de procurer des faclités pour l'emploi de l'électricité à des usages agricoles, le Ministère de l'Agriculture a fait ıde cette règle la base des projets de loi qu'il a déposés en ce qui concerne tes usines hydrauliques sur les cours d'eau non navigables.

Les diverses mesures qui viennenl d'être énumérées facilitent les développements des emplois de l'électricité d'une façon en quelque sorte indirecte. L'intervention de l'Administration s'est exercée plus directement par l'organisation de coopératives de production et de distribution d'énergie. L'Etat a joué à leur égard un rôle bienfaisant à un double point de vue : d une part, en leúr prêtant son concours au point de vue financier ; d'autre part, en les aidant au point de vue technique par la collaboration du service des améliorations agricoles. C'est ainsi qu'ont été. constituées diverses installations coopératives menées à bien dans les départements de l'Aisne, de l'Aubc, de la Côte-d'Or et de la Marne. Parmi ces coopératives quelques-unes achètent le courant à ides Sociétés qui corisentent à le vendre à un prix acceptable, d'autres, au contraire, ont pour objel à la fois la production el la distribution de l'énergie créée par une centrale.

Après avoir fait remarquer qu'il résulte de l'ensemble des indications précédentes que les efforts de l'Administration se sont exercés dans les sens les plus divers pour généraliser les utilisations ide l'énergie électrique en agriculture, M. DABat expose comment on pourra réaliser de nouveaux progrès.

Les Sociétés de distribution négligeaient complètement, il y a quelques années, la clientèle des agriculteurs. Vendre de l'électricité aux prix largement rémunérateurs que pernettent certains emplois comme l'éclairage, n'abaisser les tarifs qu'en faveur de très gros consommateurs comme les entreprises de transports ou certaines industries : telle était la doctrine appliquée. Mais les concessionnaires ont fini par reconnaître que s'il était justifié d'avoir au début de leur exploitation recherché avant tout la 